\title{
Performance Evolution of MAC Layer in IEEE 802.11g Networks
}

\author{
Tayyeba Minhas, Satish Anamalamudi, Xu Ning, and Minglu Jin
}

\begin{abstract}
The popularity of IEEE 802.11 networks comes with its low cost, high data rate and seamless handoff. With heavily utilized infrastructure based wireless networks, number of users are increasing rapidly which makes available unlicensed spectrum band more congested. Moreover, most of the IEEE 802.11 standards $(\mathrm{b} / \mathrm{g} / \mathrm{n})$ operate on $2.4 \mathrm{GHz}$ which has subject to interference with other services like Bluetooth, sensor networks and cordless phones. Hence, the performance of IEEE 802.11 MAC should be enhanced with respect to interference and spectral scarcity. In general, IEEE 802.11 networks operate on either DCF (Distributed co-ordination function) or PCF (Point co-ordination function) mode. This research paper mainly focuses on PCF mode IEEE 802.11g standard and explains the necessity of amendment in terms of frequency, throughput, net bit rate, maximum indoor range and maximum output range. OPNET Simulator is used to calculate network load, throughput and medium access delay. Moreover, calculated performance metrics are compared with different user probability for IEEE 802.11g networks.
\end{abstract} load.

Index Terms-IEEE 802.11g, MAC, throughput, network

\section{INTRODUCTION}

Wireless communications has seen an eminent growth and will certainly continue its outstanding developments due to the support of new interactive real-time multimedia applications with advanced microelectronic devices. IEEE 802.11 specifications are set of wireless standards that specify an 'over-the-air' interface between wireless hosts and a base station or access point. An 802.11 LAN is based on a cellular architecture where the space is divided into cells. Each cell (called Basic Service Set, or BSS, in the 802.11 nomenclature) is controlled by a Base Station (called Access Point or, in short, AP). The functional overview of IEEE $802.11 \mathrm{~g}$ networks is clearly shown in Fig. 1. Mobile station (STA) refers to the device that contains an IEEE 802.11 medium access control(MAC) and physical layer (PHY) interface to the wireless medium (WM). Access Point (AP) provides access to the distribution services via the wireless medium (WM) for associated stations [1]. The Basic Service Set (BSS) is the basic building block of an IEEE 802.11 wireless LAN which contains group of stations. Basic Service Area (BSA) is defined as conceptual area

Manuscript received December 9, 2012; revised March 1, 2013.

T. Minhas and $\mathrm{N}$. $\mathrm{Xu}$ are with School of computer science Wuhan University of technology, Wuhan, China (email: knoor50@yahoo.com, xuning@whut.edu.cn).

S. Anamalamudi and M. L. Jin are with School of Information and Communication Engineering Dalian university of Technology, Dalian, China (email:satishnaidu80@gmail.com, mljin@dlut.edu.cn) within which, the members of the Basic Service Set may communicate. The entire interconnected Wireless LAN, including heterogeneous cells, their respective base stations(AP) and the Distribution System, is seen as a single 802 network to the upper layers of the OSI model and is known in the Standard as Extended Service Set (ESS)[2]. The rest of the paper is organized as follows. Section.II explains about related works with pros and cons. Section.III clearly describes about IEEE 802.11g PCF (Point Coordination Function) with related performance metrics. Section.IV explains about experimental results in OPNET simulator. Section.V ends up with conclusion and future work.

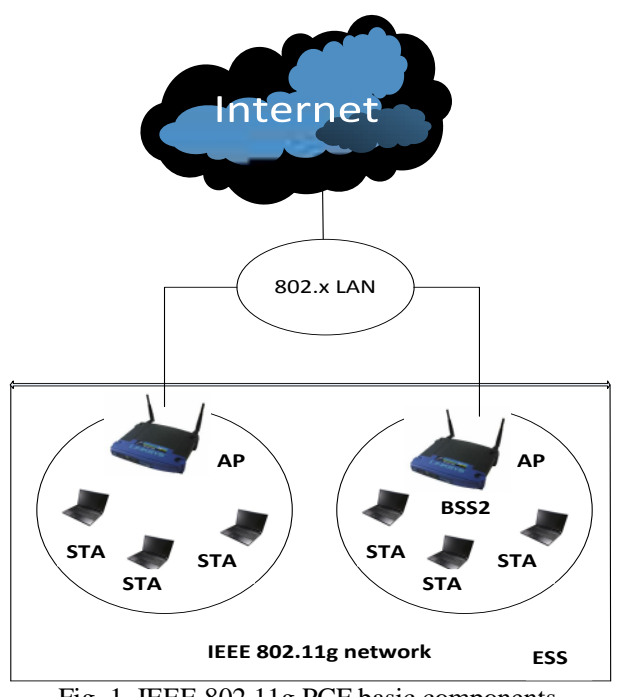

Fig. 1. IEEE 802.11g PCF basic components.

\section{RELATED WORKS}

In 1997, Institute of Electrical and Electronic Engineers (IEEE) had proposed a new communication medium through IEEE 802.11.The official 802.11 supports up to 2-Mbps which is very slow to handle high data. In 1999, amendment to IEEE 802.11 called $802.11 \mathrm{~b}$ standard had been released to support high data rate $(11 \mathrm{Mbps})$ in $2.4 \mathrm{GHz}$ spectrum band Even though $802.11 \mathrm{~b}$ supports high data rate, it is subject to interference with Bluetooth, Wireless sensor networks and cordless phones. Hence, interference might be possible with other services which results performance degradation in IEEE 802.11b networks.

Subsequently, IEEE 802.11a was developed along with IEEE $802.11 \mathrm{~b}$ networks which supports up to 54-Mbps in 5$\mathrm{MHz}$ spectrum band without interference with other services [3]. But, usage of 5-MHz spectrum band will leads to short range signals which results short range data transmission. Moreover, it is relatively costlier than $802.11 \mathrm{~b}$ networks. Hence, hybrid of both 802.11 b and 802.11 a called $802.11 \mathrm{~g}$ networks are proposed to support high data rate with long 
range data transmission without interference to other services. Hence, most of the users are trying to connect with IEEE 802.11g network. Since, 802.11g [4]-[6] uses same 2.4 $\mathrm{GHz}$ like $802.11 \mathrm{~b}$ there is a possibility to have interference with other services whenever, the network load is very high.
This paper focus on throughput and performance comparison for different user probability in IEEE $802.11 \mathrm{~g}$ networks. Moreover, our research extends to compare media access delay and network load for different user probability.

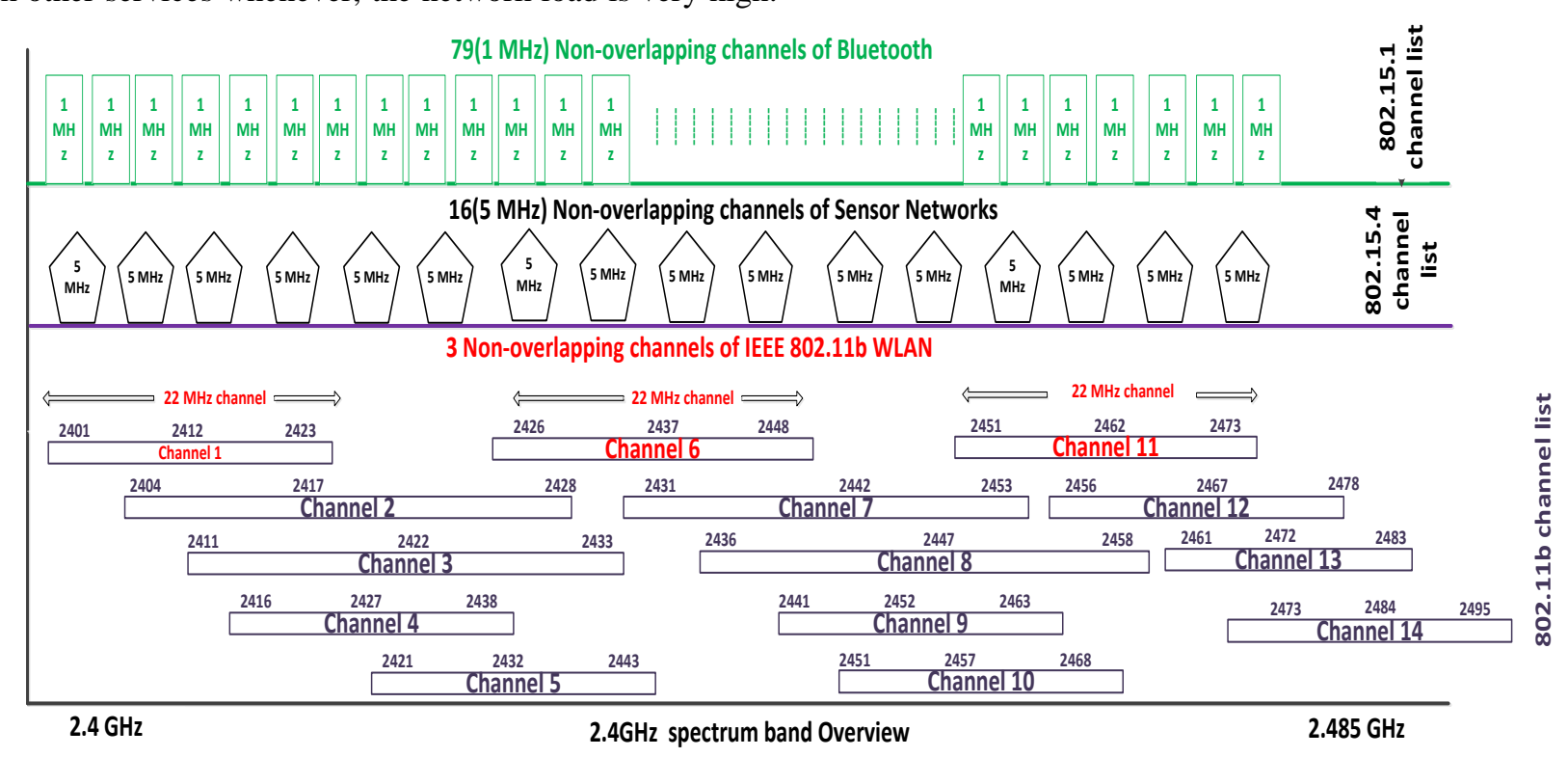

Fig. 2. IEEE $802.11 \mathrm{~b}$ interference scenario with other Communication Services.

III. PROTOCOL DESCRIPTION AND PERFORMANCE METRICS

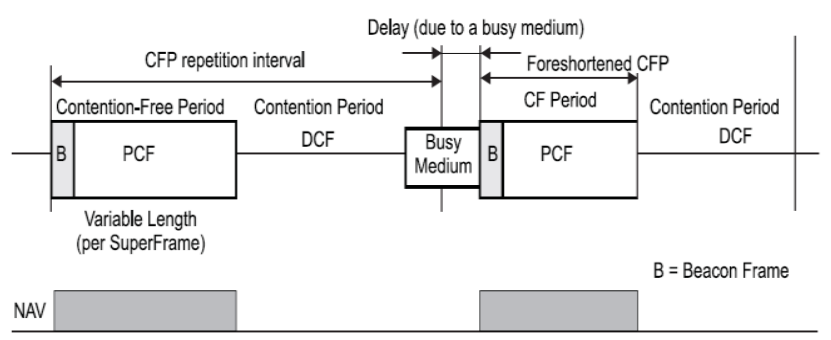

Fig. 3. PCF Operation In IEEE $802.11 \mathrm{~g}$ Networks.

This paper is restricted to PCF mode of IEEE $802.11 \mathrm{~g}$ networks and the functional overview is clearly explained in Fig. 3. PCF is built on top of the DCF (Distribute Coordination Function) which provides contention free frame transfer through Point Coordinator (PC) [7]. In PCF mode, Access point acts as a central authoritative agent for channel allocation, authentication and authorization. The PCF is using the virtual carrier -sense mechanism which is added by the access priority mechanism. The PCF should have the distributed information within beacon management frames to get the control of the medium by setting (NAV) network allocation vector in STA it means that all the frames using PCS have the small (IFS) inter frame space which is smaller than the IFS to those frames which are using DCF. Point Co-coordinator which resides in the Access Point, polls individual stations for a period of time called the Contention Free Period (CFP). This is followed by a Contention Period (CP) during which stations communicate directly under the control of the DCF using CSMA/CA protocol [8]. CFP repetition interval (CFPrate) which resides within point coordination function is used to determine frequency of contention-free transfer. Usually access point initiates a contention free transmission (CFP) through beacon message
(B) shown in Fig. 3. Whenever, Point Coordination Function is executed, timeslots are divided into Contention Free Periods (CFP), wherein the AP gives transmission opportunity to the stations and Contention Periods (CP), where the DCF is executed.

Once Short inter frame sequence (SIFS) is completed with PCF operation; a poll packet is combined with data packet at base station. Upon the reception, STA1 acknowledges to access point for receiving data packet. Once, the AP receives ACK data from STA1 it combines POLL packet and data packet for STA2. The complete cycle of contention free period completes with CFP End (CE) control message. Maximum duration for contention free polling is shown in (1).

$$
\begin{aligned}
& \mathrm{CFPMax}_{\text {Duration }}=\left(\text { Beacon }_{\text {period }} \times \mathrm{DTIM}_{\text {period }} \times \mathrm{CFP}_{\text {Rate }}\right. \\
& \mathrm{CFPMax}_{\text {Duration }}=\mathrm{MaxMPDU}_{\text {Time }}+\left(2 \times \mathrm{aSIFS}_{\text {Time }}\right)+\left(2 \times \mathrm{aSlot}_{\mathrm{Ti}}\right. \\
& \text { me })+\left(8 \times \mathrm{ACK}_{\text {Size }}\right)
\end{aligned}
$$

\section{A. Performance Metrics}

Throughput: In radio communication networks, throughput or network throughput is defined as the average rate of successful message delivery over a communication channel. Data may be delivered through physical or logical link, or pass through a certain network node. Throughput is usually measured in bits per second (bit/sec). System throughput or Aggregate throughput is defined as sum of data rates that are delivered to all STA's within communication range. Throughput directly depends on control message overhead and congestion. As the control message overhead and collision increases overall throughput will be decreased.

\section{B. Network Load}

Bandwidth offered by an Access Point is shared among all STA's within its coverage area. Throughput of an Access 
Point increases in proportion to the amount of packet traffic load added by all connected STA's provided that the overall traffic load does not exceed the capacity of the AP. When AP's workload exceeds or approaches its capacity, the throughput does not increase further. This phenomenon is called channel Saturation or congestion. In general, highly overloaded AP may simply reject new association requests. If an AP is not overloaded then it grants association request from STA's based on work-load status [9]. The request can be granted only when the predicted load level after the association does not exceed some predefined threshold. A simple way to increase overall system throughput is to deploy additional APs covering the same region, in anticipation that heavy traffic load can be distributed among multiple APs. Unfortunately, as each STA independently selects an AP to camp on, STA's may be associated with few APs while other APs remain idle. Consequently, the traffic load is not fairly shared by APs. This problem motivates load-balancing protocols for IEEE 802.11 networks. The design goal is to make AP association loadaware, preventing possible associations with congested AP [10]. The ultimate goal is to increase overall system throughput. In our approach, we calculate network load for different traffic with different STA's to calculate saturation point.

\section{Media Access Delay}

Whenever, data is being transmitted from sender to receiver there is delay due to resource scarcity. Usually there are different types of delays in communication network namely processing delay, queuing delay, transmission delay and propagation delay. Hence, time taken to receive data from sender to receiver is shown in (2).

Traffic intensity $=\mathrm{La} / \mathrm{R}$

$$
\text { Delay }_{\text {Total }}=\mathrm{D}_{\text {Proc }}+\mathrm{D}_{\text {queue }}+\mathrm{D}_{\text {trans }}+\mathrm{D}_{\text {prop }}
$$

\section{where}

$L=$ Payload length (bits);

$a=$ Average packet arrival rate

$R=$ link bandwidth(bps)

$L a / R \sim 0$ : Queuing delay: small

$L a / R$-> 1: Queuing delay: large.

$L a / R>1$ : Queuing delay: Infinite

In general, queuing delay can be calculated through traceroute with corresponding server address.

\section{SiMULATION RESULTS}

OPNET network simulator is use to create network and generate performance metrics for different scenarios. This section will provides the combine effects of load, Media access delay and throughput for the four scenarios namely, WLAN2STA, WLAN4STA, WLAN8STA and WLAN16STA.

\section{A. Gregate Throughput}

Fig. 5. shows the Wireless LAN throughput (bit/sec) for four scenarios WLAN2STA, WLAN4STA, WLAN8STA and WLAN16STA. In each scenario, the number of Stations are increasing from two STA's to 16 STA. As shown in Fig.
5, channel throughput is higher in 16 STA scenario compared with other.

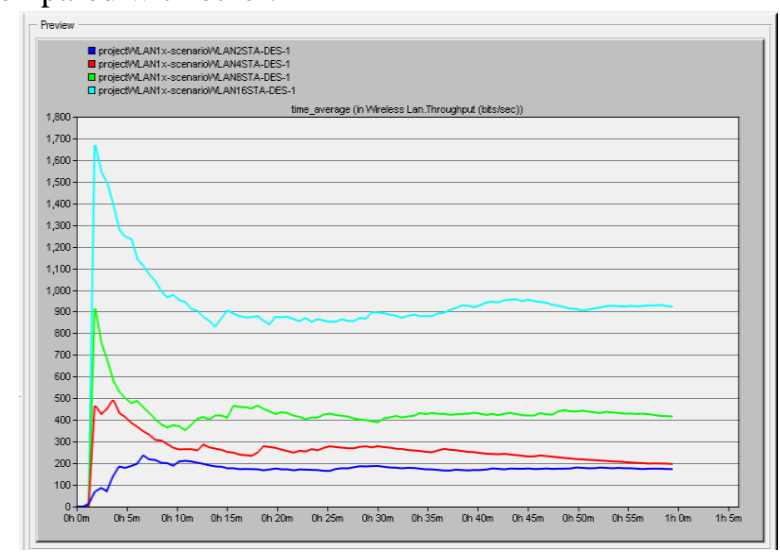

Fig. 5. Throughput comparison of various scenario's in OPNET

\section{B. Media Access Delay}

Fig. 6, shows that when there are only two wireless LAN STAs then STA that want to access the media it don't need to wait longer, but when the wireless LAN stations going increase then the station that want to access media has to wait for longer time. When the STA'S are 16 then the Media Access delay for one STA that want access media is much longer compared with other scenarios.

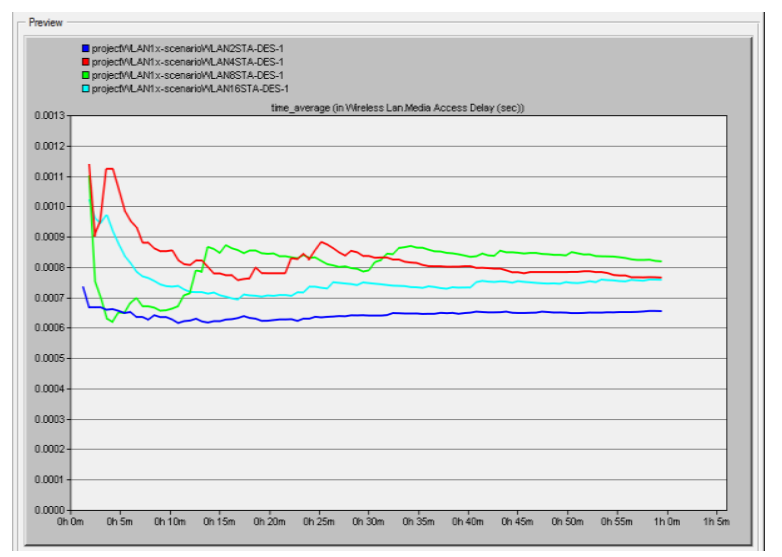

Fig. 6. Media access Delay comparison of various scenario's in OPNET.

\section{Network Load}

Fig. 7 explains about network load for various scenarios. It shows that whenever, we increase the number of wireless LAN station then the load on Access Pont will be increases.

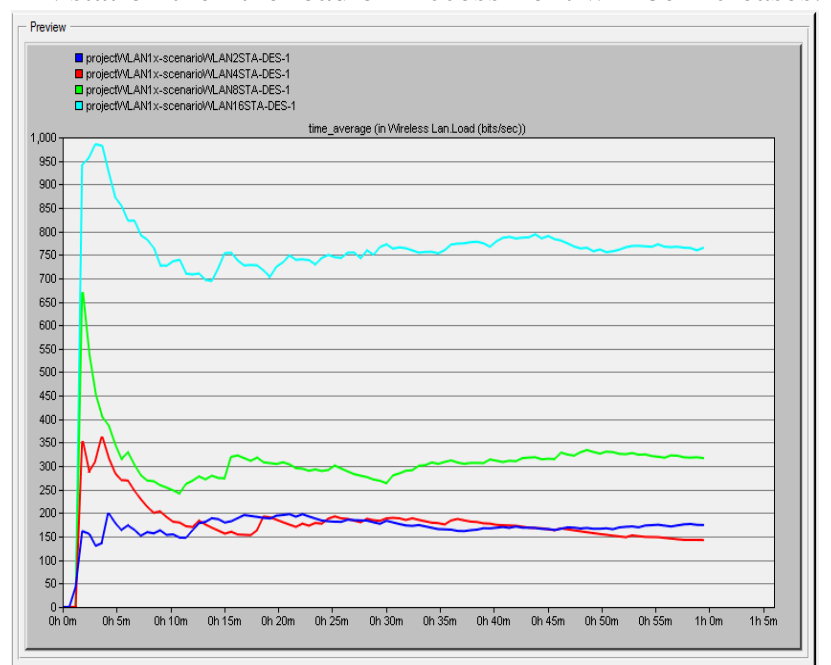

Fig. 7. Network load comparison of various scenario's in OPNET. 
This is because the available channel bandwidth is finite and leads to saturation. Hence, effective network load algorithms have to propose to minimize the throughput and performance degradation due to higher network load in heavy traffic access points.

\section{CONCLUSION}

In this paper, three different performance metrics for MAC PCF based IEEE 802.11g networks are calculated and compared with different scenarios in OPNET network simulator. Based on the simulation results, it is clear that throughput of the wireless networks depends on network load. That is for specific network load achievable throughput is very high. Whenever, the network load is higher than available channel bandwidth then achievable throughput will be degraded because of collisions. Hence, it is crucial to define threshold network threshold for efficient channel utilization. Moreover, media access delay is calculated and compared with different scenarios. Based on experimental results it is clear that media access delay will be higher for larger STA networks.

\section{ACKNOWLEDGMENT}

The authors would like to thank for anonymous reviewers for their invaluable comments and suggestions.

\section{REFERENCES}

[1] S. V. Saliga "An introduction to IEEE 802.11 wireless LANs," Radio Frequency Integrated Circuits (RFIC) Symposium, Digest of Papers, 2000, IEEE, pp.11-14, 2000, doi: 10.1109/RFIC.2000.854406.

[2] L. Loyola, T. Kumagai. K. Nagata. S. Otsuki, and S. Aikawa, "Multi-channel wireless LAN mesh architecture with DCF-based inter-AP communication and idle channel search packet forwarding," in Proc. IEEE Global Telecommunications Conference, vol. 6, pp. 3279-3284, 2-2 Dec. 2005 doi: 10.1109/GLOCOM.2005.1578381.

[3] P. Mahasukhon, M. Hempel, C. Song, and H. Sharif, "Comparison of Throughput Performance for the IEEE 802.11a and 802.11g Networks," AINA '07. 21st International Conference on Advanced Information Networking and Applications, pp.792-799, 21-23 May 2007, doi: 10.1109/AINA.2007.46

[4] S.-C. Wang, Y.-M. Chen, T.-H. Lee, and A. Helmy, "Performance evaluations for hybrid IEEE $802.11 \mathrm{~b}$ and $802.11 \mathrm{~g}$ wireless networks," in Proc. 24th IEEE International Performance, Computing, and Communications Conference, pp. 111-118, 7-9 April 2005, doi: 10.1109/PCCC.2005.1460529.

[5] T. L. Wang and H. H. Refai, "Network performance analysis on IEEE $802.11 \mathrm{~g}$ with different protocols and signal to noise ratio values," in Proc. Second IFIP International Conference on Wireless and Optical Communications Networks, pp. 29- 33, 6-8 March 2005, doi: 10.1109/WOCN.2005.1435983.

[6] M. Vucinic, B. Tourancheau, and A. Duda, "Simulation of a backward compatible IEEE 802.11g network: Access delay and throughput performance degradation," in Proc. 2012 Mediterranean Conference on Embedded Computing (MECO), pp. 190-195, 19-21 June 2012.

[7] B. Sikdar, "An Analytic Model for the Delay in IEEE 802.11 PCF MAC-Based Wireless Networks," IEEE Transactions on Wireless Communications, vol. 6, no.4, pp. 1542-1550, April 2007, doi: 10.1109/TWC.2007.348350
[8] D. D. Vergados and D. J. Vergados, "Synchronization of multiple access points in the IEEE 802.11 point coordination function," IEEE 60th Vehicular Technology Conference, vol. 2, pp. 1073- 1077, 2629 Sept. 2004, doi: 10.1109/VETECF.2004.1400186

[9] L. H. Yen, T. T. Yeh, and K. H. Chi, "Load Balancing in IEEE 802.11 Networks," Internet Computing, IEEE, vol. 13, no. 1, pp. 5664, Jan.-Feb. 2009, doi: 10.1109/MIC.2009.11.

[10] P. C. Ng and S. C. Liew, "Offered load control in IEEE 802.11 multi-hop ad-hoc networks," 2004 IEEE International Conference on Mobile Ad-hoc and Sensor Systems, pp. 80- 89, 25-27 Oct. 2004, doi: 10.1109/MAHSS.2004.1392078.

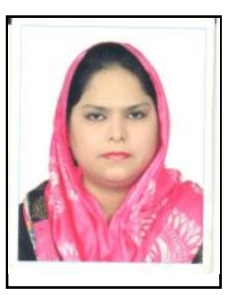

Tayyeba Minhas is currently a $\mathrm{PhD}$ candidate in School of Computer Science and Technology, Wuhan University of Technology, Wuhan, China. Her research interests includes database and datwarehouse management systems and MAC protocol design in Mobile ad-hoc networks.

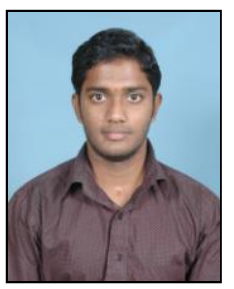

Satish Anamalamudi is currently a $\mathrm{PhD}$ candidate in School of Information and Communication Engineering, Dalian University of Technology, Dalian, China. His research interests includes Common control channel design for MAC and routing protocols in Cognitive radio ad-hoc networks, Performance enhancement of TCP in mobile and cognitive radio ad-hoc networks.

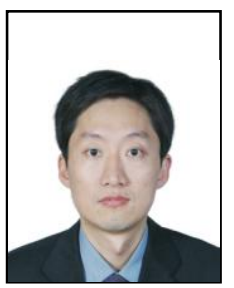

Ning $\mathrm{Xu}$ received his $\mathrm{Ph}$. D. degree in electronic science and technology from the University of Electronic Science and Technology of China in 2003. Later, he was a postdoctoral fellow with Tsinghua University from 2003 to 2005 . Currently, he is a professor at the Computer Science Department of Wuhan University of Technology. Dr. Xu's research interests include computer-aided design of VLSI circuits and systems, computer architectures, data mining, and highly combinatorial optimization algorithms. He has published over 50 research papers and led over 5 research projects in these areas. He is a senior member of China Computer Federation and the Chinese Institute of Electronics.

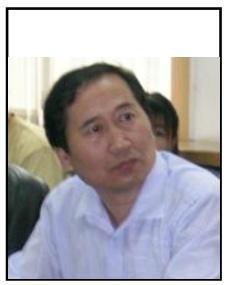

Minglu Jin is a professor in the School of Electronics \& Information Engineering at Dalian University of Technology, china. He received the Ph.D. and M.Sc. degrees from Beihang University,china, the B.Eng. degree from University of Science \& Technology of China. He was a Visiting scholar in the Arimoto Lab. at Osaka University, Japan from 1987 to 1988 . He was a Research Fellow in Radio \& Broadcasting Research Lab. at ETRI, Korea from 2001 to 2004. Professor JIN's research interests are in the general areas of signal processing and communications systems. Specific current interests are nonsinusoidal function theory and its applications, power amplifier linearization, radio over fiber, nulling antenna techniques. 